\title{
MicroRNAs regulate signaling pathways in osteogenic differentiation of mesenchymal stem cells (Review)
}

\author{
SHUPING PENG ${ }^{1,2}$, DAN GAO $^{1,2}$, CHENGDE GAO $^{3}$, PINGPIN WEI $^{1,2}$ MAN NIU $^{1,2}$ and CIJUN SHUAI ${ }^{3}$ \\ ${ }^{1}$ Hunan Provincial Tumor Hospital and The Affiliated Tumor Hospital of Xiangya School of Medicine, \\ Central South University, Changsha, Hunan 410013; ${ }^{2}$ School of Basic Medical Science, Cancer Research Institute, \\ Central South University, Changsha, Hunan $410078 ;{ }^{3}$ State Key Laboratory of High Performance Complex Manufacturing, \\ Central South University, Changsha, Hunan 410083, P.R. China
}

Received June 17, 2015; Accepted April 18, 2016

DOI: $10.3892 / \mathrm{mmr} .2016 .5335$

\begin{abstract}
Osteogenesis is a complex multi-step process involving the differentiation of mesenchymal stem cells (MSCs) into osteoblast progenitor cells, preosteoblasts, osteoblasts and osteocytes, and the crosstalk between multiple cell types for the formation and remodeling of bone. The signaling regulatory networks during osteogenesis include various components, including growth factors, transcription factors, micro (mi)RNAs and effectors, a number of which form feedback loops controlling the balance of osteogenic differentiation by positive or negative regulation. miRNAs have been found to be important regulators of osteogenic signaling pathways in multiple aspects and multiple signaling pathways. The present review focusses on the progress in elucidating the role of miRNA in the osteogenesis signaling networks of MSCs as a substitute for bone implantation the the field of bone tissue engineering. In particular, the review classifies which miRNAs promote or suppress the osteogenic process,
\end{abstract}

Correspondence to: Professor Cijun Shuai, State Key Laboratory of High Performance Complex Manufacturing, Central South University, 932 South Yuelu Mountain Road, Changsha, Hunan 410083, P.R. China

E-mail: shuai@csu.edu.cn

Abbreviations: OCN, osteocalcin; OPN, osteopotin; ALP, alkaline phosphatase; hBMSCs, human bone marrow mesenchymal stem cells; SCAPs, apical papilla stem cells; BMP2, bone morphogenetic protein 2; hADMSCs, human adipose tissue-derived mesenchymal stem cells; hADSCs, human adipose-derived stem cells; hAT-MSC, human adipose tissue-derived MSC; PDLSCs, periodontal ligament stem cells; OSX, osteoblast-specific transcription factor; FABP4, fatty acid binding protein 4; KLF4, kruppel-like factor 4; TNF $\alpha$, tumor necrosis factor $\alpha$; HDAC6, histone deacetylase 6; APC, adenomatous polyposis coli; TCF3, transcription factor 3; SIRT1 silent, information regulator 1

Key words: osteogenic differentiation, microRNAs, mesenchymal stem cells, signaling and summarizes which signaling pathway these miRNAs are involved in. Improvements in knowledge of the characteristics of miRNAs in osteogenesis provide an important step for their application in translational investigations of bone tissue engineering and bone disease.

\section{Contents}

1. Introduction

2. miRNAs promote the osteogenic differentiation of mesenchymal stem cells

3. miRNAs suppress the osteogenesis of mesenchymal stem cells

4. Conclusion

\section{Introduction}

MicroRNAs (miRNAs) are an extensive family of small (18-24 nucleotides), single-stranded non-coding RNAs, which regulate gene expression in mammalian cells through binding to the seed sequences of the 3'-untranslated region (UTR) of target mRNA sequences and mediate the degradation of mRNA in the RNA-induced silencing complex $(1,2)$. miRNAs repress translation by decreasing stability through targeting specific mRNA targets. Each miRNA regulates numerous mRNAs, and miRNAs are involved in various cellular processes, including proliferation, differentiation, cell cycle, invasion and apoptosis (3-6). The alterations in their expression levels may lead to human diseases, including cardiovascular disease and cancer (7-10). The formation of bone by osteoblast cells and their primary functional activities involve a series of multiple signals, including bone morphogenic protein (BMP), Wnt ligands, Notch ligands, hormones and growth factors, including transforming growth factor (TGF) and tumor necrosis factor (TNF) and cytokines. In addition to these factors, tissue-specific transcription factors and co-factors mediate the expression of genes for the biosynthesis and mineralization of bone matrix, and the remodeling and formation of bone (11-15). Mesenchymal stem cells (MSCs) are induced to differentiate into preosteoblasts, which are regulated by the 


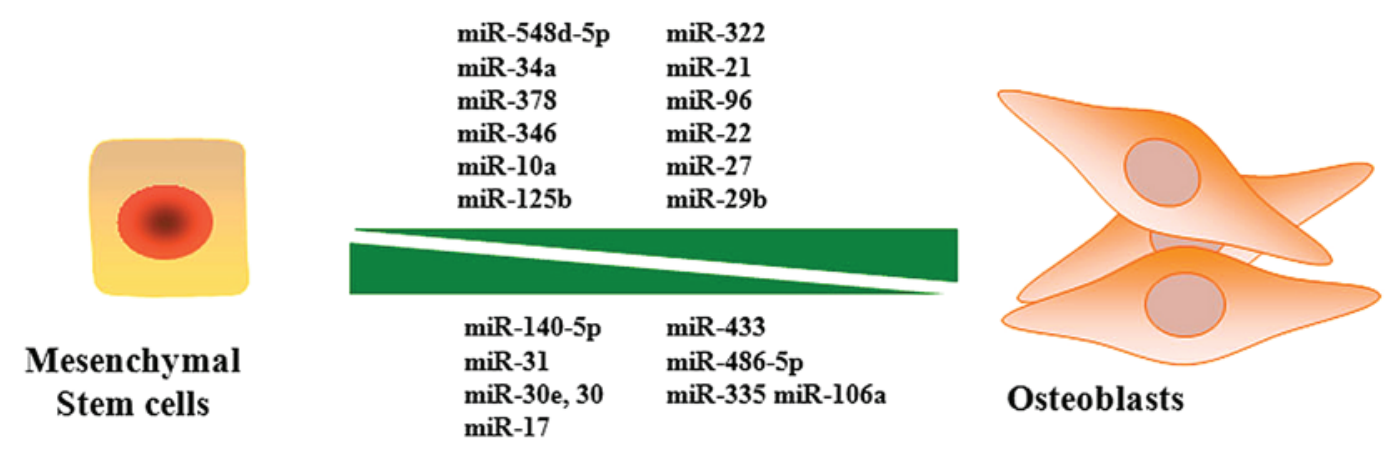

Figure 1. miRNAs control the osteogenic differentiation of mesenchymal stem cells into osteoblasts. The microRNA above the green box indicates that they promote the osteogenic differentiation of mesenchymal stem cells into osteoblasts; the microRNAs listed below the green box indicates that they inhibit the osteogenic differentiation of mesenchymal stem cell into osteoblasts. miRNA/miR, microRNA.

signaling cascades initiated by the various signals, including cytokine stimuli. Pre-osteoblasts differentiate into osteoblasts, which is controlled by gene expression affected by chromatin modifiers, transcription factors or miRNA alterations (16). Osteoblasts produce bone matrix, which mineralizes into bone tissue, and osteoblasts are engulfed in the matrix during bone formation. The entire process is complex and well organized by the signaling network.

Experimental evidence shows that miRNAs are critical for stem cell activities, particularly in the stemness maintenance or direct differentiation into lineage cells $(7,8)$. To date, several studies have examined the functions of miRNAs in MSCs $(17,18)$. Certain miRNAs have been shown to regulate the process of differentiation of MSCs into different cell lineages using high-through gene expression microprofiling assays. This has led to the specific targets of these miRNAs being determined, and the roles of miRNAs are gradually being elucidated (Fig. 1).

\section{2. miRNAs promote the osteogenic differentiation of mesenchymal stem cells}

2.1. BMP signaling. BMP-induced osteogenic differentiation is well known $(19,20)$. Hupkes $\mathrm{M}$ et al reported that the expression of miRNA regulated the terminal differentiation of $\mathrm{C} 2 \mathrm{C} 12$ myoblasts via lineage-specific changes. It was found that the overexpression of miR-378 enhances alkaline phosphatase (ALP) activity, calcium deposition and the mRNA expression levels of osteogenic marker genes in the presence of BMP2 (21). miR-322, a BMP-2-downregulated miRNA, is a regulator of osteoblast differentiation. Using gain-and loss-of-function experiments of miR-322 in the $\mathrm{C} 2 \mathrm{C} 12$ mouse myoblast cell line, MC3T3-E1 mouse embryonic osteoblasts and primary murine bone marrow-derived MSCs, the overexpression of miR-322 has been demonstrated to enhance the BMP-2 response, increasing the expression of osteoblast-specific transcription factor (Osx) and other osteogenic genes. Transducer of erbB2 (Tob2) has been identified as a target of miR-322, as reporter assays revealed that miR-322 binds to the specific sequence in the 3'-UTR of Tob2. Tob2 is a negative regulator of osteogenesis, which binds to and mediates the degradation of Osx mRNA. This demonstrates a novel molecular mechanism controlling osteogenesis through the specific miR-322/Tob2 regulation of specific target mRNAs (22). miR-196a is involved in the proliferation and osteogenic differentiation of human adipose-derived stem cells (hADSCs). The overexpression of miR-196a inhibits hASC proliferation and enhances osteogenic differentiation by targeting the 3'UTR sequence of homeobox (HOX)C8 mRNA. The expression of Hoxc8 in C2C12 cells decreases the alkaline phosphatase activity induced by BMP-2. The expression of HOXC8 is decreased during the osteogenic differentiation of hADSCs, and concomitant with an increase in the level of miR-196a. Thus, inhibition of miR-196a decreases the protein levels of HOXC8 in hADSCs, and is accompanied by increased osteogenic differentiation. This indicates that miR-196a has a positive effect in osteogenic differentiation in hADSCs by repressing HOXC8 (23).

All the miRNAs promoting osteogenic differentiation are listed in Fig. 2 and Table I.

2.2. Wnt/ $\beta$-catenin signaling. Wnt/ $\beta$-catenin signaling has been well defined in the osteogenic differentiation of MSCs (24-27). miR-346 promotes the osteogenic differentiation of human bone marrow MSCs (hBMSCs) by

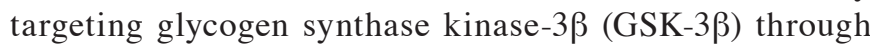
binding to the 3 '-UTR of its mRNA. The decreased GSK-3 $\beta$ results in an increase of $\beta$-catenin, which is translocated into the nucleus and activates the downstream genes of the Wnt/ $\beta$-catenin pathway. $\beta$-catenin knockdown almost completely inhibits the positive effect of miR-346 on osteogenic differentiation. Therefore, miR-346 positively regulates the osteogenic differentiation of hBMSCs through the Wnt/ $\beta$-catenin pathway (28-31). The expression of miR-27 is increased during hFOB1.19 cell (human SV40 transfected osteoblast) differentiation. The ectopic expression of miR-27 promotes the differentiation of hFOB1.19 cells by directly targeting and inhibiting the gene expression of adenomatous polyposis coli (APC). This inhibition of the expression of APC leads to the accumulation of $\beta$-catenin, which is a key protein activating Wnt signaling. This suggests that miR-27 is an important promoter of osteogenic differentiation (32). miR-218 positively regulates the osteogenesis of hADSC, directly targeting secreted frizzled-related protein 2 and dickkopf WNT signaling pathway inhibitor 2, thus enhancing $W n t / \beta$-catenin signaling progression. Mimics of the $W n t / \beta$-catenin signal increase the expression of miR-218, which forms a positive feedback loop to promote osteogenesis (33). 


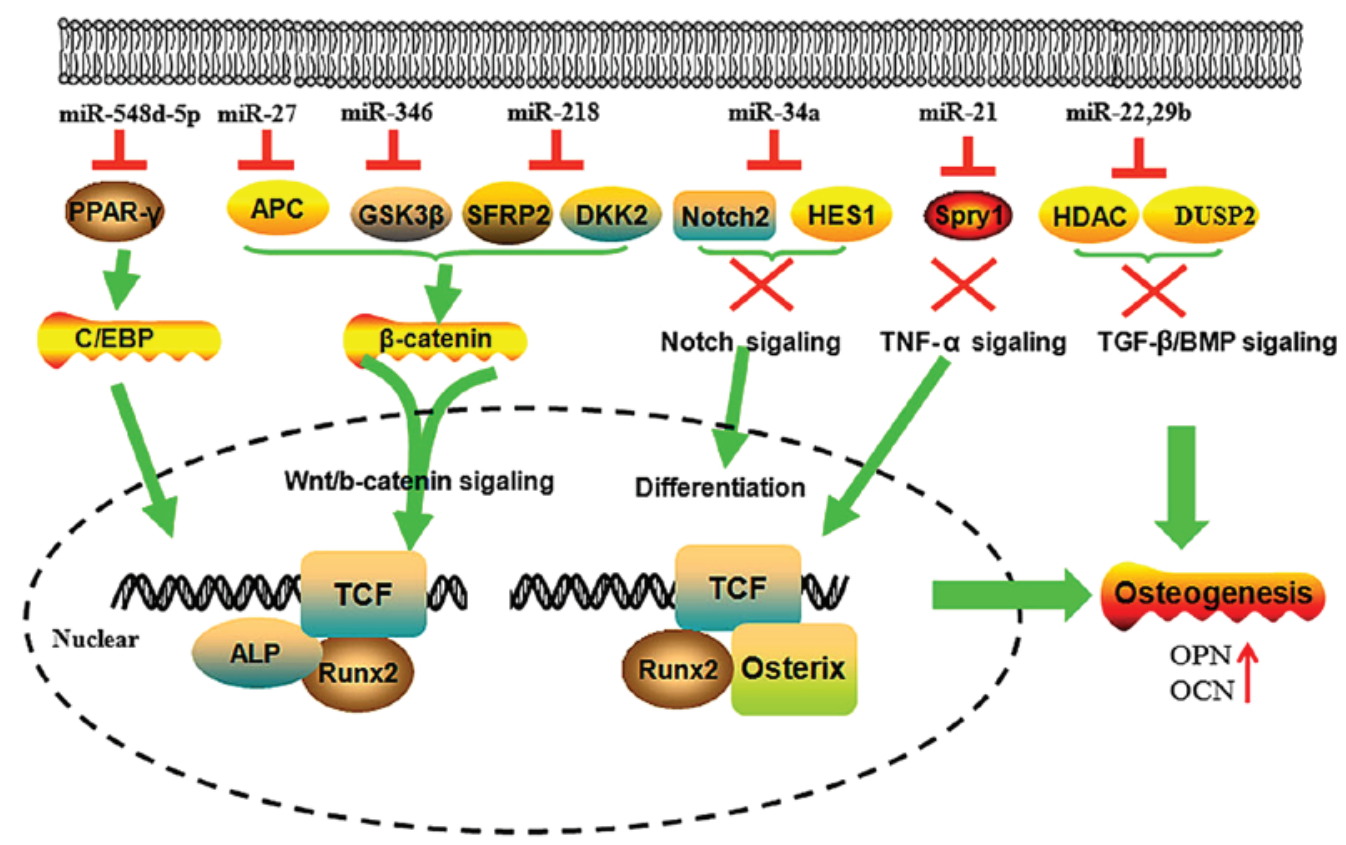

Figure 2. MiR-548, miR-27, miR-346, miR-218, miR-34a, miR-21, miR-22 and miR-29b promote the osteogenic differentiation of mesenchymal stem cells through Wnt/catenin, Notch, TNF- $\alpha$, TGF- $\beta$ /BMP signaling pathways. miR, microRNA; PPAR- $\gamma$, peroxisome proliferator-activated receptor- $\gamma$; GSK-3 $\beta$, glycogen synthase kinase-3 $\beta$; Spry1, sprouty homolog 1; HDAC, histone deacetylase; DUSP2, dual specificity phosphatase 2; APC, adenomatous polyposis coli; SFRP2, secreted frizzled-related protein 2; DKK2, dickkopf WNT signaling pathway inhibitor 2; TCF, transcription factor; Runx2, runt-related transcription factor 2; OPN, osteopontin; OCN, osteocalcin; TNF- $\alpha$, tumor necrosis factor- $\alpha$; TGF- $\beta$, transforming growth factor- $\beta$; BMP, bone morphogenetic protein.

2.3. Notch signaling. Notch Signaling is important in maintaining the undifferentiated state of MSCs (34-38). The activation of Notch signaling in apical papilla stem cells (SCAPs) inhibits cell differentiation. High expression levels of miR-34a inhibits Notch signaling by directly targeting the 3 'UTR of Notch2 and HES1 mRNA then suppressing the binding of Notch2, and HES1, then the inhibition of Notch signaling pathway, resulting in the expression of runt-related transcription factor 2 (RUNX2) and OSX, and enhancing the expression of osteocalcin (OCN). miR-34a promotes osteogenesis in SCAPs through Notch signaling by targeting Notch 2 and HES1 (39).

The upregulation of miR-10a results in increased osteogenic differentiation. miR-10a mimics significantly repress luciferase activity by the direct binding to the 3'-UTR of Kruppel-like factor 4 (KLF4). The inhibition of KLF4 in hBMSCs increases cell differentiation. Therefore, miR-10a enhances the differentiation capability of hBMSCs through the repression of KLF4 (40).

2.4. TNF- $\alpha$ signaling. MiR-21 has been confirmed to promote the osteogenic differentiation of mouse bone marrow cells by targeting Sprouty homolog 1 (Spry1), negatively regulating the osteogenic differentiation of MSCs. The expression of miR-21 partially rescues TNF- $\alpha$-impaired osteogenesis of MSCs. Inhibition of the TNF- $\alpha$ signaling pathway evidently improves bone formation and down-regulates Spryl expression, which suggests that miR-21 contributes towards bone formation through targeting Spry1 in MSCs (41).

miR-96 and miR-199a were both up-regulated during osteogenic induction of human bone marrow derived MSCs. They may function through transcription factor SRY-box 9 , and fatty acid binding protein 4 , however, the detailed molecular mechanism remains poorly understood (42).
The expression of miR-22 is increased during the process of osteogenic differentiation of hADSCs. miR-22 inhibits the expression of histone deacetylase 6 (HDAC6) by binding to the similar sequence of the 3'-UTR (43). HDAC6 deficiency results in a minor increase in trabecular bone density. Thus, miR-22 increases osteogenesis through targeting HDAC6.

miR-29b is understood to promote osteogenesis by supressing the negative regulators, including histone deacetylase 4 , TGF- $\beta 3$, activin A receptor (Type IIA), catenin beta interacting protein 1 and dual specificity phosphatase 2 , during the induction of osteogenic differentiation. These anti-osteogenic factors negatively modulate extracellular matrix excretion by differentiated osteoblasts and bone formation $(44,45)$.

\section{3. miRNAs suppress the osteogenesis of mesenchymal stem cells}

Several miRNAs directly target transcription factors, including osteopotin (OPN), OCN and RUNX2, or signaling molecules, which promote the osteogenesis of MSCs and inhibit osteogenic differentiation (Fig. 3 and Table I). miR-140-5p inhibits the osteogenic differentiation in hMSCs by directly blocking BMP2, and consequently blocking BMP signaling components and critical regulators (46).

The overexpression of miR-31 represses the osteogenesis of hMSCs by directly targeting special AT-rich sequence-binding protein 2 (SATB2), the knockdown of SATB2 by specific siRNA against SATB2 inhibits osteogenic differentiation (47). Baglio et al found an inverse trend in miRNA-target expression during osteogenic differentiation between the levels of miR-31 and OSX. The inhibition of miR-31 leads to an increase in the endogenous expression of OSX (48). Deng et al showed that 
Table I. miRNAs regulate osteogenesis during the transition of mesenchymal stem cells into osteoblasts.

\begin{tabular}{|c|c|c|c|c|c|}
\hline Function & miRNA & Target gene & Cell & Author & Refs \\
\hline \multirow[t]{17}{*}{ Promotor } & miR-34a & NOTCH2 and HES1 & human SCAPs & Sun et al (2014) & $(37)$ \\
\hline & miR-378 & None validated & C2C12, myoblasts & Hupkes et al (2014) & $(21)$ \\
\hline & miR-346 & GSK-3 $\beta$ & hBMSCs & Wang et al (2013) & $(26)$ \\
\hline & & & & Westendorf et al (2004) & $(27)$ \\
\hline & & & & Logan et al (2004) & $(28)$ \\
\hline & & & & Gaur et al (2005) & $(29)$ \\
\hline & miR-10a & KLF4 & hMSCs & Li et al (2013) & $(38)$ \\
\hline & miR-322 & Tob2 & mBMSCs & Gamez et al (2013) & $(39)$ \\
\hline & $\operatorname{miR}-21$ & Spry1 & MSCs & Yang et al (2013) & $(40)$ \\
\hline & miR-96 & SOX9, aggrecan and FABP4 & hMSCs & Laine et al (2012) & $(41)$ \\
\hline & miR-22 & HDAC6 & hADMSCs & Huang et al (2012) & $(42)$ \\
\hline & $\operatorname{miR}-27$ & APC & hFOB 1.19 cells & Wang and $\mathrm{Xu}(2010)$ & $(30)$ \\
\hline & $\operatorname{miR}-218$ & SFRP2 and DKK2 & hASCs & Zhang et al (2014) & (31) \\
\hline & $\operatorname{miR}-29 b$ & COL1A1, COL5A3 & osteoblasts & Huang et al (2012) & $(42)$ \\
\hline & & and COL4A2 & & Crane and Cao (2014) & (44) \\
\hline & miR-196a & $\mathrm{HOXC8}$ & hASCs & Kim et al (2009) & $(45)$ \\
\hline & $\operatorname{miR}-140-5 p$ & BMP2 & hMSCs & Hwang et al (2014) & $(46)$ \\
\hline \multirow[t]{11}{*}{ Suppressor } & $\operatorname{miR}-31$ & SATB2 and OSX & hMSCs, BMSCs & Xie et al (2014) & $(47)$ \\
\hline & & & & Baglio et al (2013) & $(48)$ \\
\hline & & & & Deng et al (2013) & $(49)$ \\
\hline & miR-30e & TCF & $\begin{array}{l}\mathrm{C} 3 \mathrm{H} 10 \mathrm{~T} 1 / 2 \\
\text { pre-adipocyte } \\
\text { 3T3-L1 }\end{array}$ & Wang et al (2013) & $(50)$ \\
\hline & miR-30 & Smad1 and Runx2 & & Wu et al (2012) & $(51)$ \\
\hline & $\operatorname{miR}-17$ & TCF3 and Smurf1 & PDLSCs & Liu et al (2011) & $(52)$ \\
\hline & miR-17-5p, miR-106a & BMP2 & hADSCs & Li et al (2013) & $(53)$ \\
\hline & $\operatorname{miR}-433$ & Runx2 & $\mathrm{C} 3 \mathrm{H} 10 \mathrm{~T} 1 / 2$ & Kim et al (2013) & $(54)$ \\
\hline & $\operatorname{miR}-486-5 p$ & SIRT1 & hAT-MSCs & Kim et al (2012) & $(55)$ \\
\hline & miR-335 & RUNX2 & hMSCs & Tome et al (2011) & $(56)$ \\
\hline & $\operatorname{miR}-135 b$ & IBSP and OSX & USSCs & $\begin{array}{l}\text { Schaap-Oziemlak } \\
\text { et al (2010) }\end{array}$ & $(57)$ \\
\hline
\end{tabular}

miR/miRNA, microRNA; MSCs, mesenchymal stem cells; hBMSCs, human bone marrow MSCs; SCAPs, apical papilla stem cells; hADMSCs, human adipose tissue-derived MSCs; hADSCs, human adipose-derived stem cells; hAT-MSCs, human adipose tissue-derived MSCs; PDLSCs, periodontal ligament stem cells; USSCs, unrestricted somatic stem cells; GSK-3 $\beta$, glycogen synthase kinase-3 $\beta$; KLF4, kruppel-like factor 4; Tob2, transducer of erbB2; Spry1, sprouty homolog 1; FABP4, fatty acid binding protein 4; SOX9, SRY-box 9; HDAC6, histone deacetylase 6; APC, adenomatous polyposis coli; SFRP2, secreted frizzled-related protein 2; DKK2, dickkopf WNT signaling pathway inhibitor 2; COL, collagen; HOX, homeobox; BMP2, bone morphogenetic protein 2; SATB2, special AT-rich sequence-binding protein 2; TCF, transcription factor; Smad1, small mothers against decapentaplegic 1; Runx2, runt-related transcription factor 2; Smurf1, Smad ubiquitin regulatory factor 1; SIRT1, silent information regulator 1; IBSP, integrin-binding sialoprotein; OSX, osteoblast-specific transcription factor.

the expression of miR-31 decreased progressively in BMSC cultures during differentiation. The upregulation of miR-31 significantly reduces the expression levels of osteogenic transcription factors, OPN, bone sialoprotein, OSX and OCN. The inhibition of miR-31 markedly decreases the activity of ALP and inhibits osteogenesis. These results suggest that an miR-31/SATB2 axis is involved in the osteogenic differentiation of BMSCs (49).

miR-30 family members are also important regulators during the biomineralization process. The overexpression of miR-30e stimulates adipocyte formation and inhibits osteoblast differentiation from marrow stromal cells. Low-density lipoprotein receptor-related protein 6 (LRP6) is one of the critical co-receptors for Wnts. Blocking LRP6 in 3T3-L1 cells downregulates $\beta$-catenin/T-cell factor transcriptional activity and enhances osteogenic differentiation. miR-30 has been reported to target the LRP6 directly and inhibit the expression of LRP6 expression. Thus, miR-30e also controls osteogenesis in periodontal ligament stem cell (PDLSCs) by targeting LRP6 and affecting canonical Wnt/ $\beta$-catenin signaling (50). There is also evidence that miR-30 family members negatively regulate BMP-2-induced osteoblast differentiation by targeting Small 


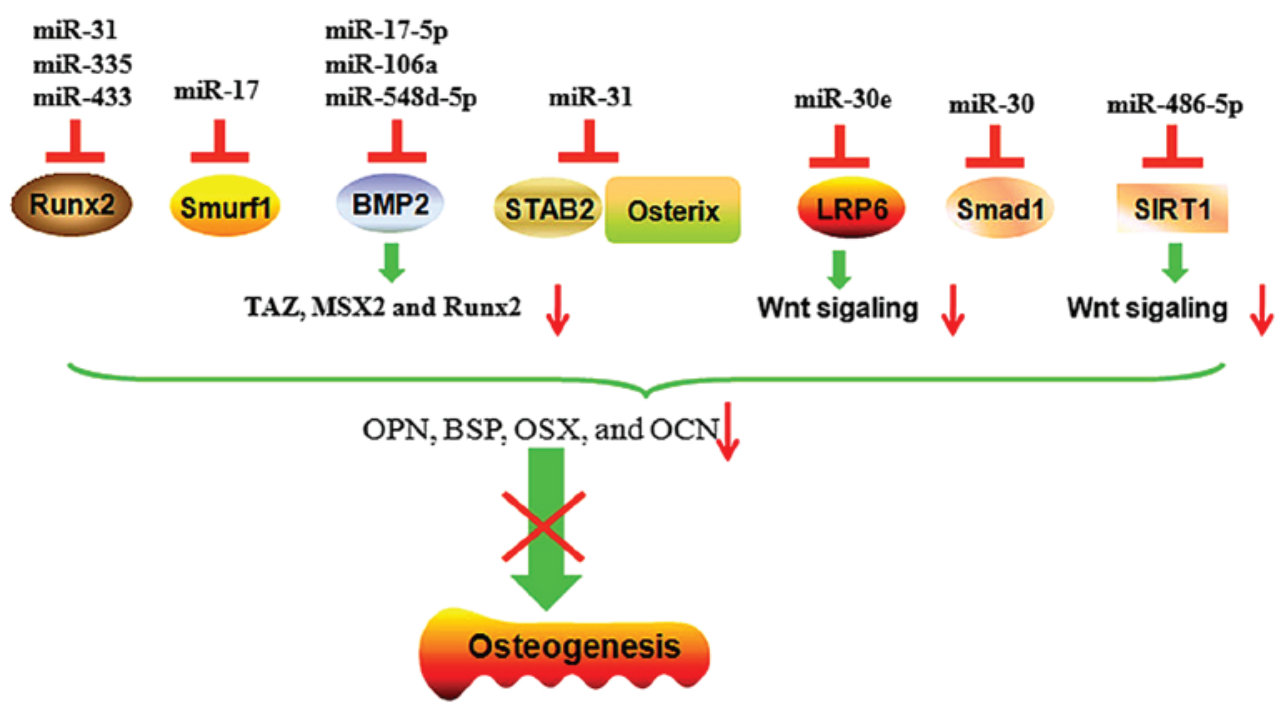

Figure 3. MicroRNAs suppress the osteogenic differentiation of mesenchymal stem cells through targeting transcription factors RUNX2, Smuf1, Osterix or BMP protein. miR, microRNA; Runx-2, runt-related transcription factor 2; BMP, bone morphogenetic protein; STAB2, stabilin 2; LRP6, lipoprotein receptor-related protein 6; Smad1, small mothers against decapentaplegic 1; SIRT1, silent information regulator 1; Smurf1, Smad ubiquitin regulatory factor 1; TAZ, transcriptional coactivator with PDZ-binding motif; MSX2, Msh homeobox 2; OPN, osteopontin; BSP, bone sialoprotein; OSX, osteoblast-specific transcription factor; OCN, osteocalcin.

mothers against decapentaplegic (Smad)1 and RUNX2. As Smad1 and RUNX2 are key positive transcription factors in osteogenic differentiation, miR-30 family members negatively regulate the osteogenic differentiation through Smad1 and RUNX2 (51).

Liu et al (52) elucidated that transcription factor 3 (TCF3) enhances osteogenesis in PDLSCs. miR-17 can inhibit this effect by suppressing the target gene, TCF3. Furthermore, the overexpression of TCF3 blocks the effect of miR-17 on the modulation of Wnt signaling (52). Inflammation promotes the downregulation of miR-17 and the subsequent increased expression of Smad ubiquitin regulatory factor 1 (Smurf1), which is an important negative regulator of osteogenic differentiation in MSCs by promoting the degradation of Smurf1-mediated osteoblast-specific factors. Smurf1 has been confirmed as a direct target of miR-17 in PDLSCs using a luciferase reporter assay (52). Li H et al revealed that miR-17-5p and miR-106a have dual functions in hADSC fate, which can promote adipogenesis and inhibit osteogenesis. The inhibitory effects of miR-17-5p and miR-106a are due to directly targeting BMP2, and subsequently decreased osteogenic TAZ, MSX2 and RUNX2 protein levels (53).

During osteoblastic differentiation, the overexpression of Estrogen-related receptor $\gamma(E R R \gamma)$ or miR-433 inhibits the expression levels of osteogenic marker genes including RUNX2 and ALP. miR-433 directly targets three binding sites on the 3'-UTR of RUNX2 mRNA, and decreases the levels of the RUNX2 transcript in C3H10T1/2 cells. Anti-miR-433 recovers the ERR $\gamma$-suppressed expression of RUNX2 and activity of ALP. ERR $\gamma$ is able to upregulate the levels of miR-433 expression and further enhance the inhibitory role in osteogenic differentiation. This evidence demonstrates that miR-433 suppresses BMP2-induced osteogenic differentiation by targeting RUNX2 C3H10T1/2 cells (54).

The overexpression of miR-486-5p inhibits osteogenic differentiation of ADSCs.miR-486-5pregulates the expression of silent information regulator 1 (SIRT1), a major regulator of longevity and metabolic disorders. SIRT1 also has an important role in the osteogenic process by targeting FOXO3A and then upregulating the levels of RUNX2 promoter activity. miR-486-5p inhibits the expression of SIRT1 through binding to the 3'-UTR region of SIRT1 mRNA (55). The overexpression of miR-335 in hMSCs derived from bone marrow, adipose tissue and articular cartilage inhibits their osteogenic and adipogenic potential. The expression of miR-335 in hMSCs is upregulated by the activated canonical Wnt signaling pathway. It has also been confirmed that RUNX2 is a direct target of miR-335. These results suggest that the downregulation of miR-335 is critical for the acquisition of MSC phenotype differentiation into osteoblasts (56). The overexpression of miR-135b downregulates the osteogenic differentiation of unrestricted somatic stem cells through targeting the key osteogenic factors, integrin-binding sialoprotein and OSX, inhibiting the process of osteogenesis (57).

\section{Conclusion}

There is substantial evidence and experimental data confirming that miRNAs have multi-dimensional roles in the induction of MSCs into osteoblasts. miRNAs function at all stages of osteoblast differentiation by inhibiting the negative regulators of signaling pathways operating in these cells (16). They also have direct and indirect effects on phenotype development through the promotion or inhibition of positive or negative transcription factors in signaling pathways, which are involved in complex regulatory networks. This indicates an important technique, by which the network of osteogenic differentiation can be regulated through miRNAs. Nicotine can alter the expression of miRNA and reduce human adult stem cell regenerative potential, demonstrating that miRNAs function in the network $(58,59)$.

Currently, only a limited number of miRNAs, identified through in vitro expression profiling of bone cells undergoing 
differentiation programs, have been characterized for their functional activity and known targets. Numerous miRNAs are associated with bone development, which can be utilized in new bone formation and the therapy of osteoporosis or bone malignant diseases. However, the crosstalk of multiple types of molecules in the regulatory network of the osteogenic differentiation process is not well-defined. LncRNA and circular RNAs which are emerging as novel regulators in the cellular processes, including osteogenesis, require further investigation (60).

\section{Acknowledgements}

This study was supported by funds from The Natural Science Foundation of China (grant nos. 81572577 and 81428018), the Program for New Century Excellent Talents in University (grant no. NCET-12-0544), the Open-End Fund for the Valuable and Precision Instruments of Central South University, Hunan Provincial Natural Science Foundation of China (grant no. 14JJ1006) and the Project of Innovation-driven Plan of Central South University (grant no. 2016CX023).

\section{References}

1. Brennecke J, Hipfner DR, Stark A, Russell RB and Cohen SM: Bantam encodes a developmentally regulated microRNA that controls cell proliferation and regulates the proapoptotic gene hid in drosophila. Cell 113: 25-36, 2003.

2. Bartel DP and Chen CZ: Micromanagers of gene expression: The potentially widespread influence of metazoan micrornas. Nat Rev Genet 5: 396-400, 2004

3. Hyun J, Wang S, Kim J, Rao KM, Park SY, Chung I, Ha CS, Kim SW, Yun YH and Jung Y: MicroRNA-378 limits activation of hepatic stellate cells and liver fibrosis by suppressing Gli3 expression. Nat Commun 7: 10993, 2016

4. Cui Y, Han J, Xiao Z, Chen T, Wang B, Chen B, Liu S, Han S, Fang Y, Wei J, et al: The miR-20-Rest-Wnt signaling axis regulates neural progenitor cell differentiation. Sci Rep 6:23300, 2016.

5. Gao Y, Chen L, Song H, Chen Y, Wang R and Feng B: A double-negative feedback loop between E2F3b and miR-200b regulates docetaxel chemosensitivity of human lung adenocarcinoma cells.Oncotarget: Mar 25, 2016 (Epub ahead of print).

6. Ma L, Ma S, Zhao G, Yang L, Zhang P, Yi Q and Cheng S: miR-708/LSD1 axis regulates the proliferation and invasion of breast cancer cells. Cancer Med 5: 684-692, 2016.

7. Kim VN: Microrna biogenesis: Coordinated cropping and dicing. Nat Rev Mol Cell Biol 6: 376-385, 2005.

8. Lu J, Getz G, Miska EA, Alvarez-Saavedra E, Lamb J, Peck D, Sweet-Cordero A, Ebert BL, Mak RH, Ferrando AA, et al: Microrna expression profiles classify human cancers. Nature 435 : 834-838, 2005.

9. Thum T and Mayr M: Review focus on the role of microRNA in cardiovascular biology and disease. Cardiovasc Res 93: 543-544, 2012.

10. Van Rooij E and Olson EN: Microrna therapeutics for cardiovascular disease: Opportunities and obstacles. Nat Rev Drug Discov 11: 860-872, 2012.

11. Oryan A, Alidadi S, Moshiri A and Bigham-Sadegh A: Bone morphogenetic proteins: A powerful osteoinductive compound with non-negligible side effects and limitations. Biofactors 40: 459-481, 2014.

12. Yuan Z, Li Q, Luo S, Liu Z, Luo D, Zhang B, Zhang D, Rao P and $\mathrm{Xiao} \mathrm{J}$ : PPAR $\gamma$ and Wnt signaling in adipogenic and osteogenic differentiation of mesenchymal stem cells. Curr Stem Cell Res Ther 11: 216-225, 2016.

13. Ongaro A, Pellati A, Bagheri L, Rizzo P, Caliceti C, Massari L and De Mattei M: Characterization of notch signaling during osteogenic differentiation in human osteosarcoma cell line MG63. J Cell Physiol: Mar 4, 2016 (Epub ahead of print).
14. Chen $\mathrm{YJ}$, Yao $\mathrm{CC}$, Huang $\mathrm{CH}$, Chang $\mathrm{HH}$ and Young $\mathrm{TH}$ : Hexosamine-induced TGF- $\beta$ signaling and osteogenic differentiation of dental pulp stem cells are dependent on $\mathrm{N}$-acetylglucosaminyltransferase V. Biomed Res Int 2015: 924397, 2015.

15. Marupanthorn K, Tantrawatpan C, Tantikanlayaporn D, Kheolamai P and Manochantr S: The effects of TNF- $\alpha$ on osteogenic differentiation of umbilical cord derived mesenchymal stem cells. J Med Assoc Thai 98 Suppl 3: S34-S40, 2015.

16. Lian JB, Stein GS, van Wijnen AJ, Stein JL, Hassan MQ, Gaur T and Zhang Y: microRNA control of bone formation and homeostasis. Nat Rev Endocrinol 8: 212-227, 2012.

17. Van Wijnen AJ, van de Peppel J, van Leeuwen JP, Lian JB, Stein GS, Westendorf JJ, Oursler MJ, Im HJ, Taipaleenmäki H, Hesse E, et al: Microrna functions in osteogenesis and dysfunctions in osteoporosis. Curr Osteoporos Rep 11: 72-82, 2013.

18. Dong S, Yang B, Guo H and Kang F: microRNAs regulate osteogenesis and chondrogenesis. Biochem Biophys Res Commun 418: 587-591, 2012.

19. Lamplot JD, Qin J, Nan G, Wang J, Liu X, Yin L, Tomal J, Li R, Shui W, Zhang H, et al: Bmp9 signaling in stem cell differentiation and osteogenesis. Am J Stem Cells 2: 1-21, 2013.

20. Peng Y, Kang Q, Cheng H, Li X, Sun MH, Jiang W, Luu HH, Park JY, Haydon RC and He TC: Transcriptional characterization of bone morphogenetic proteins (BMPs)-mediated osteogenic signaling. J Cell Biochem 90: 1149-1165, 2003.

21. Hupkes M, Sotoca AM, Hendriks JM, van Zoelen EJ and Dechering KJ: Microrna mir-378 promotes BMP2-induced osteogenic differentiation of mesenchymal progenitor cells. BMC Mol Biol 15: 1, 2014.

22. Wagner ER, Zhu G, Zhang BQ, Luo Q, Shi Q, Huang E, Gao Y, Gao JL, Kim SH, Rastegar F, et al: The therapeutic potential of the Wnt signaling pathway in bone disorders. Curr Mol Pharmacol 4: 14-25, 2011

23. Kim JH, Liu X, Wang J, Chen X, Zhang H, Kim SH, Cui J, Li R, Zhang W, Kong Y, et al: Wnt signaling in bone on and its therapeutic potential for bone diseases. Ther Adv Musculoskelet Dis 5: 13-31, 2013.

24. Marcellini S, Henriquez JP and Bertin A: Control of osteogenesis by the canonical Wnt and Bmp pathways in vivo: Cooperation and antagonism between the canonical Wnt and BMP pathways as cells differentiate from osteochondroprogenitors to osteoblasts and osteocytes. Bioessays 34: 953-962, 2012.

25. Krause $U$ and Gregory CA: Potential of modulating Wnt signaling pathway toward the development of bone anabolic agent. Curr Mol Pharmacol 5: 164-173, 2012.

26. Wang Q, Cai J, Cai XH and Chen L: Mir-346 regulates osteogenic differentiation of human bone marrow-derived mesenchymal stem cells by targeting the Wnt/ $\beta$-catenin pathway. PLoS One 8: e72266, 2013.

27. Westendorf JJ, Kahler RA and Schroeder TM: Wnt signaling in osteoblasts and bone diseases. Gene 341: 19-39, 2004.

28. Logan CY and Nusse R: The Wnt signaling pathway in development and disease. Annu Rev Cell Dev Biol 20: 781-810, 2004.

29. Gaur T, Lengner CJ, Hovhannisyan H, Bhat RA, Bodine PV, Komm BS, Javed A, van Wijnen AJ, Stein JL, Stein GS and Lian JB: Canonical WNT signaling promotes osteogenesis by directly stimulating Runx2 gene expression. J Biol Chem 280: 33132-33140, 2005.

30. Wang T and Xu Z: Mir-27 promotes osteoblast differentiation by modulating Wnt signaling. BiochemBiophys Res Commun 402: 186-189, 2010.

31. Zhang WB, Zhong WJ and Wang L: A signal-amplification circuit between miR-218 and $\mathrm{Wnt} / \beta$-catenin signal promotes human adipose tissue-derived stem cells osteogenic differentiation. Bone 58: 59-66, 2014

32. Li Y, Li SQ, Gao YM, Li J and Zhang B: Crucial role of Notch signaling in osteogenic differentiation of periodontal ligament stem cells in osteoporotic rats. Cell Biol Int 38: 729-736, 2014.

33. Shimizu T, Tanaka T, Iso T, Doi H, Sato H, Kawai-Kowase K, Arai $\mathrm{M}$ and Kurabayashi M: Notch signaling induces osteogenic differentiation and mineralization of vascular smooth muscle cells role of Msx2 gene induction via Notch-RBP-Jk signaling. Arterioscler Thromb Vasc Biol 29: 1104-1111, 2009.

34. Shimizu T, Tanaka T, Iso T, Matsui H, Ooyama $\mathrm{Y}$, Kawai-Kowase K, Arai M and Kurabayashi M: Notch signaling pathway enhances bone morphogenetic protein 2 (BMP2) responsiveness of Msx2 gene to induce osteogenic differentiation and mineralization of vascular smooth muscle cells. J Biol Chem 286: 19138-19148, 2011. 
35. Shindo K, Kawashima N, Sakamoto K, Yamaguchi A, Umezawa A, Takagi M, Katsube K and Suda H: Osteogenic differentiation of the mesenchymal progenitor cells, Kusa is suppressed by Notch signaling. Exp Cell Res 290: 370-380, 2003.

36. Ugarte F, Ryser M, Thieme S, Fierro FA, Navratiel K, Bornhäuser $\mathrm{M}$ and Brenner S: Notch signaling enhances osteogenic differentiation while inhibiting adipogenesis in primary human bone marrow stromal cells. Exp Hematol 37: 867-875, 2009

37. Sun F, Wan M, Xu X, Gao B, Zhou Y, Sun J, Cheng L, Klein OD, Zhou $X$ and Zheng L: Crosstalk between miR-34a and notch signaling promotes differentiation in apical papilla stem cells (SCAPs). J Dent Res 93: 589-595, 2014 (Epub ahead of print)

38. Li J, Dong J, Zhang ZH, Zhang DC, You XY, Zhong Y, Chen MS and Liu SM: Mir-10a restores human mesenchymal stem cell differentiation by repressing KLF4. J Cell Physiol 228: 2324-2336, 2013.

39. Gamez B, Rodriguez-Carballo E, Bartrons R, Rosa JL and Ventura F: microRNA-322 (miR-322) and its target protein Tob2 modulate osterix (osx) mrna stability. J Biol Chem 288 14264-14275, 2013.

40. Yang N, Wang G, Hu C, Shi Y, Liao L, Shi S, Cai Y, Cheng S, Wang X, Liu Y, et al: Tumor necrosis factor $\alpha$ suppresses the mesenchymal stem cell osteogenesis promoter miR-21 in estrogen deficiency-induced osteoporosis. J Bone Miner Res 28: 559-573, 2013.

41. Laine SK, Alm JJ, Virtanen SP, Aro HT and Laitala-Leinonen TK: microRNAs miR-96, miR-124 and miR-199a regulate gene expression in human bone marrow-derived mesenchymal stem cells. J Cell Biochem 113: 2687-2695, 2012.

42. Huang S, Wang S, Bian C, Yang Z, Zhou H, Zeng Y, Li H, Han Q and Zhao RC: Upregulation of miR-22 promotes osteogenic differentiation and inhibits adipogenic differentiation of human adipose tissue-derived mesenchymal stem cells by repressing HDAC6 protein expression. Stem Cells Dev 21: 2531-2540, 2012

43. Li Z, Hassan MQ, Jafferji M, Aqeilan RI, Garzon R, Croce CM, van Wijnen AJ, Stein JL, Stein GS and Lian JB: Biological functions of miR-29b contribute to positive regulation of osteoblast differentiation. J Biol Chem 284: 15676-15684, 2009.

44. Crane JL and Cao X: Bone marrow mesenchymal stem cells and TGF- $\beta$ signaling in bone remodeling. J Clin Invest 124 : 466-472, 2014.

45. Kim YJ, Bae SW, Yu SS, Bae YC and Jung JS: Mir-196a regulates proliferation and osteogenic differentiation in mesenchymal stem cells derived from human adipose tissue. J Bone Miner Res 24 816-825, 2009

46. Hwang S, Park SK, Lee HY, Kim SW, Lee JS, Choi EK, You D, Kim CS and Suh N: Mir-140-5p suppresses BMP2-mediated osteogenesis in undifferentiated human mesenchymal stem cells. FEBS Lett 588: 2957-2963, 2014.

47. Xie Q, Wang Z, Bi X, Zhou H, Wang Y, Gu P and Fan X: Effects of miR-31 on the osteogenesis of human mesenchymal stem cells. Biochem Biophys Res Commun 446: 98-104, 2014.

48. Baglio SR, Devescovi V, Granchi D and Baldini N: microRNA expression profiling of human bone marrow mesenchymal stem cells during osteogenic differentiation reveals osterix regulation by miR-31. Gene 527: 321-331, 2013.
49. Deng Y, Wu S, Zhou H, Bi X, Wang Y, Hu Y, Gu P and Fan X: Effects of a miR-31, Runx2 and Satb2 regulatory loop on the osteogenic differentiation of bone mesenchymal stem cells. Stem Cells Dev 22: 2278-2286, 2013

50. Wang J, Guan X, Guo F, Zhou J, Chang A, Sun B, Cai Y, Ma Z, Dai C, Li X and Wang B: Mir-30e reciprocally regulates the differentiation of adipocytes and osteoblasts by directly targeting low-density lipoprotein receptor-related protein 6 . Cell Death Dis 4: e845, 2013.

51. Wu T, Zhou H, Hong Y, Li J, Jiang X and Huang H: miR-30 family members negatively regulate osteoblast differentiation. J Biol Chem 287: 7503-7511, 2012.

52. Liu Y, Liu W, Hu C, Xue Z, Wang G, Ding B, Luo H, Tang L, Kong $\mathrm{X}$, Chen $\mathrm{X}$, et al: miR-17 modulates osteogenic differentiation through a coherent feed-forward loop in mesenchymal stem cells isolated from periodontal ligaments of patients with periodontitis. Stem Cells 29: 1804-1816, 2011.

53. Li H, Li T, Wang S, Wei J, Fan J, Li J, Han Q, Liao L, Shao C and Zhao RC: miR-17-5p and miR-106a are involved in the balance between osteogenic and adipogenic differentiation of adipose-derived mesenchymal stem cells. Stem Cell Res 10: 313-324, 2013.

54. Kim EJ, Kang IH, Lee JW, Jang WG and Koh JT: miR-433 mediates ERR $\gamma$-suppressed osteoblast differentiation via direct targeting to Runx2 mRNA in C3H10T1/2 cells. Life Sci 92: 562-568, 2013.

55. Kim YJ, Hwang SH, Lee SY, Shin KK, Cho HH, Bae YC and Jung JS: miR-486-5p induces replicative senescence of human adipose tissue-derived mesenchymal stem cells and its expression is controlled by high glucose. Stem Cells Dev 21: 1749-1760, 2012.

56. Tome M, López-Romero $\mathrm{P}$, Albo C, Sepúlveda JC, Fernández-Gutiérrez B, Dopazo A, Bernad A and González MA: miR-335 orchestrates cell proliferation, migration and differentiation in human mesenchymal stem cells. Cell Death Differ 18: 985-995, 2011.

57. Schaap-Oziemlak AM, Raymakers RA, Bergevoet SM, Gilissen C, Jansen BJ, Adema GJ, Kögler G, le Sage C, Agami R, van der Reijden BA and Jansen JH: microRNA hsa-miR-135b regulates mineralization in osteogenic differentiation of human unrestricted somatic stem cells. Stem Cells Dev 19: 877-885, 2010.

58. Ng TK, Carballosa CM, Pelaez D, Wong HK, Choy KW, Pang CP and Cheung HS: Nicotine alters microRNA expression and hinders human adult stem cell regenerative potential. Stem Cells Dev 22: 781-790, 2013.

59. Chen HC, Lee YS, Sieber M, Lu HT, Wei PC, Wang CN, Peng HH, Chao AS, Cheng PJ, Chang SD, et al: microRNA and messenger RNA analyses of mesenchymal stem cells derived from teeth and the Wharton jelly of umbilical cord. Stem Cells Dev 21: 911-922, 2012.

60. Dong R, Du J, Wang L, Wang J, Ding G, Wang S and Fan Z: Comparison of long noncoding RNA and mRNA expression profiles in mesenchymal stem cells derived from human periodontal ligament and bone marrow. Biomed Res Int 2014: 317853, 2014. 